\title{
CONTROLLING OSCILLATOR COHERENCE BY MULTIPLE DELAY FEEDBACK
}

\author{
Denis S. Goldobin ${ }^{1,2, *}$ And Elizaveta V. Shklyaeva ${ }^{2}$
}

\begin{abstract}
We consider the implementation of a weak feedback with two delay times for controlling the coherence of both deterministic chaotic and stochastic oscillators. This control strategy is revealed to allow one to decrease or enhance the coherence, which is quantified by the phase diffusion constant, by $2-3$ orders of magnitude without destruction of the chaotic regime, which is by an order of magnitude more than one can achieve with a single delay time. Within the framework of the phase reduction, which is a rough approximation for the chaotic oscillators and rigorous for the stochastic ones, an analytical theory of the effect is constructed.
\end{abstract}

Mathematics Subject Classification. 34H10, 93B52, 60G20.

Received September 25, 2020. Accepted November 11, 2020.

\section{INTRODUCTION}

One of the key characteristics of oscillatory systems is coherence, understood as the constancy of the oscillation frequency. Coherence determines quality of clocks, lasers, electric generators, etc., and the susceptibility of oscillators to synchronization and, therefore, control [27]. The latter is as well relevant for chaotic systems where one can introduce a phase-like variable $\varphi$, which grows by $2 \pi$ for each revolution of the system. Coherence can be quantified by means of the phase diffusion constant

$$
D \equiv \lim _{t \rightarrow+\infty} \frac{\left\langle(\varphi(t)-\varphi(0)-\langle\dot{\varphi}\rangle t)^{2}\right\rangle}{t}
$$

where $\langle\ldots\rangle$ indicates averaging.

The Pyragas' delayed-feedback control method was originally suggested for the suppression of chaotic regimes and the stabilization of an unstable periodic orbit of a given period [29, 30]. On the contrast to that, the employment of a weak delayed feedback allows one to change the diffusion constant by a few orders of magnitude without destruction of the fine structure of chaos in theory [17] and also to achieve a well pronounced effect in experimental works [5]. Most effective suppression or enhancement of the phase diffusion can be achieved when the ratio of delay time to the mean natural oscillation period (more strictly, Poincaré recurrence time) is integer or half-integer. The employment of two feedback terms with different delay times provides better

Keywords and phrases: Oscillation coherence, phase diffusion, multiple delay feedback.

${ }^{1}$ Institute of Continuous Media Mechanics, Ural Branch of RAS, Perm 614013, Russia.

2 Theoretical Physics Department, Perm State University, Perm 614990, Russia.

* Corresponding author: denis.goldobin@gmail.com

(C) The authors. Published by EDP Sciences, 2021

This is an Open Access article distributed under the terms of the Creative Commons Attribution License (https://creativecommons.org/licenses/by/4.0), which permits unrestricted use, distribution, and reproduction in any medium, provided the original work is properly cited. 
plasticity and efficiency of controlling system properties compared to the case of a single feedback [1-3]. In contrast to $[13,14,26]$, we consider the situations where delay times are incommensurable.

Additionally to the benefits of plasticity and efficiency, there is a physical motivation for the interest to the case of two delay feedbacks. Many physical systems possess an inherent feedback with delay (e.g., [9, 16, 20, 31]); for such systems, introducing a second feedback loop is a natural and simple strategy for controlling the dynamics. The efficiency of the employment of a second feedback loop in such systems is invigorated by the similarity of the mechanisms of the impact on the system for both feedbacks and, therefore, the possibility of their mutual compensation in a broad range of parameters. For instance, in [9, 31], an additional feedback is used for suppression of an unwanted instability to self-modulation in the backward-wave tube. This approach turns out to be useful with other devices as well (e.g., see [20]).

Recently, the implementation of the feedback control strategy was extended to convective systems with spatial-temporal patterns [32, 33].

While one cannot construct a general analytical theory of the effect for chaotic systems, it is instructive to consider the case of stochastic oscillators. For limit-cycle oscillators an analytical theory can be constructed. Such a theory can also serve as a useful guidance for the intuition with chaotic oscillators.

In this paper, we consider the application of feedback control with two delay times to chaotic systems, using the Lorenz system as a paradigmatic example of a chaotic convective oscillator. We show, that with a two delay feedback, one can achieve a much stronger suppression/increase of the phase diffusion without destructing the chaotic regime than with a single delay. Further, the analytical theory of the effect of a two delay feedback on stochastic oscillators is constructed within the framework of the phase reduction.

\section{Chaotic oscillators: Lorenz System}

\subsection{Oscillation phase diffusion}

Let us consider the Lorenz system [22]

$$
\begin{aligned}
& \dot{x}=\sigma(y-x), \\
& \dot{y}=r x-y-x z, \\
& \dot{z}=-b z+x y+k_{1}\left[z\left(t-\tau_{1}\right)-z(t)\right]+k_{2}\left[z\left(t-\tau_{2}\right)-z(t)\right],
\end{aligned}
$$

for "typical" values of parameters $\sigma=10, r=32, b=8 / 3$ with two delay feedback terms in the $z$-variable; $k_{j}$ and $\tau_{j}$ are the coefficients and delay times of feedbacks, respectively. For this system the oscillation phase is well defined for the projection of trajectory onto the plane $\left(u=\sqrt{x^{2}+y^{2}}, z\right)$ (see Fig. 1a and, e.g., [17]):

$$
\varphi=\arctan \frac{z-z_{0}}{u-u_{0}}
$$

where the subscript " 0 " indicates the nontrivial fixed points: $u_{0}= \pm \sqrt{2} x_{0}= \pm \sqrt{2} y_{0}=\sqrt{2 b(r-1)}, z_{0}=r-1$. Strictly speaking, $\varphi$ as it is defined here is a protophase but not the genuine phase variable [21], because its growth rate within one turnover is not uniform even averagely. Nonetheless, for the quantifiers of the long-term dynamics, such as the phase diffusion which counts the regularity of the number of phase revolutions over long periods of time, the protophase and the genuine phase yield identical results.

In the upper plots of Figures $1 \mathrm{~b}$ and 2, the dependence of the phase diffusion constant on the delay times is presented. One can see, that for $k_{1}=k_{2}$ (Fig. 1b), the diffusion constant is especially strongly affected by the feedback control on the diagonal $\tau_{1}=\tau_{2}$, which corresponds to the case of a single delay feedback of double strength $k=2 k_{1,2}$. However, in the middle panels of Figures $1 \mathrm{~b}$ and 2 , one can also see that with identical positive feedback coefficients $k_{1}=k_{2}>0$ the largest Lyapunov exponent $\lambda$ on the chaotic attractor experiences much stronger shifts toward zero than with $k_{1}=-k_{2}$ or $k_{1}=k_{2}<0$. In the bottom panel of Figure $1 \mathrm{~b}$, below the bold red curves the fixed points are stable (the stability analysis for nonchaotic regimes is provided below, 

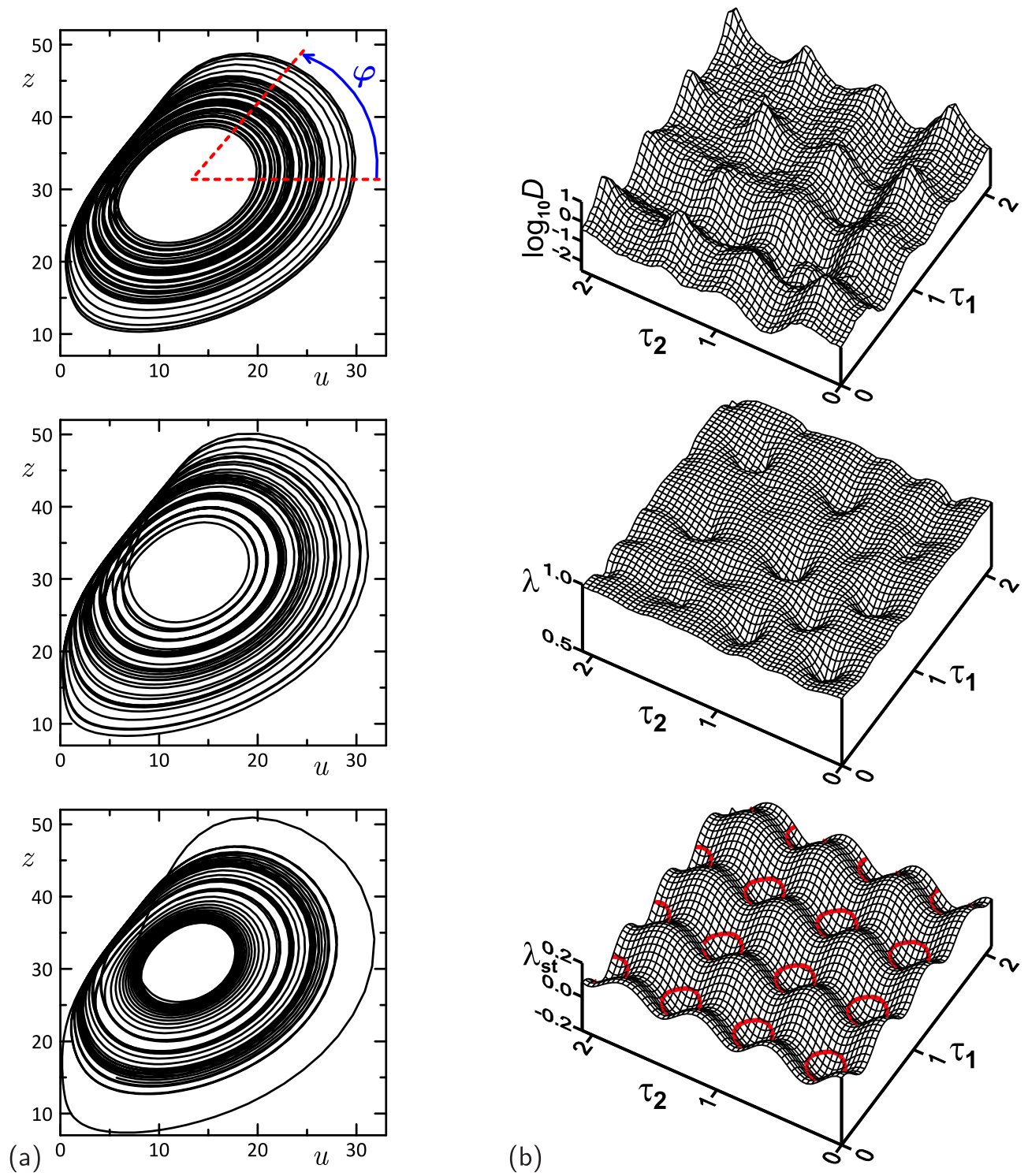

Figure 1. (a): Sample trajectories of the Lorenz system on the $(u, z)$-plane for $k_{1}=k_{2}=0.15$, $\tau_{1}=0.32, \tau_{2}=0.32,0.64,0.96$ (from top to bottom) illustrate that the phase can be well defined. (b): The phase diffusion constant $D(1.1)$ is plotted versus two delay times for the Lorenz system (2.1)-(2.3) for $k_{1}=k_{2}=0.15$ in the top panel. Middle panel: the largest Lyapunov exponent for the chaotic regime; bottom panel: the exponential growth rate of perturbations for the time-independent states $\left\{x_{0}=y_{0}= \pm \sqrt{b(r-1)}, z_{0}=r-1\right\}$, red bold contours indicate the level $\lambda_{\mathrm{st}}=0$. For the reference value of the delay time, the average Poincaré return time (roughly, "average oscillation period") of the control-free system is $T_{0} \approx 0.69$.

in Sect. 2.2). For the same absolute value of the feedback strength but $k_{1}=-k_{2}$ or $k_{1}=k_{2}<0$ (Fig. 2), the fixed points remain unstable. Therefore, with $k_{1}=k_{2}>0$ the stabilization of nonchaotic regimes and the chaos crisis occur at a lower threshold for the feedback strength than with $k_{1}=-k_{2}$ or $k_{1}=k_{2}<0$. Although for $\left|k_{1,2}\right|=0.15$ the chaotic manifold exists and remains stable for all considered values of the delay times $\tau_{1,2}$ 

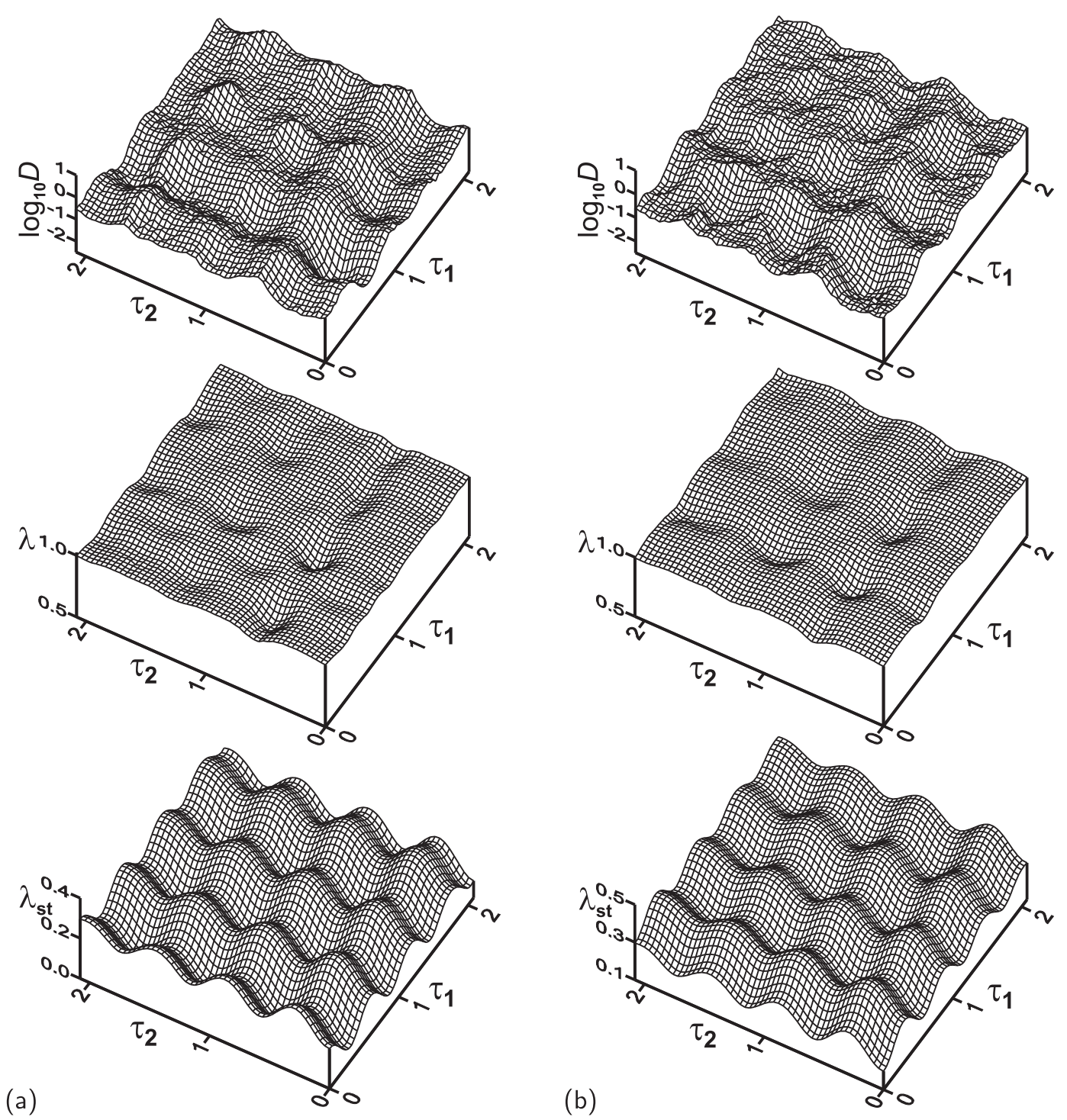

Figure 2. The phase diffusion constant $D$ is plotted versus two delay times for the Lorenz system (2.1)-(2.3) for $k_{1}=-k_{2}=0.15$ (a) and $k_{1}=k_{2}=-0.15$ (b) in the top panels. Middle panels: the largest Lyapunov exponent for the chaotic regime; bottom panels: the exponential growth rate of perturbations for the time-independent states $\left\{x_{0}=y_{0}= \pm \sqrt{b(r-1)}, z_{0}=\right.$ $r-1\}$. One can see that the time-independent states are never attracting here, in contrast to the case of $k_{1}=k_{2}=0.15$ in Figure 1.

(specifically, the Lyapunov exponent nowhere becomes lower than 0.7), the appearance of coexisting stable steady states is undesirable. First, the dynamics regime becomes sensitive to the initial conditions. Second, this multistability indicates the proximity of the crisis of chaotic regime.

Qualitatively, as one should expect, positive feedback suppresses the phase diffusion for integer $\tau_{j} / T_{0}$ (where $T_{0}$ is the average Poincaré return time of the control-free system) and increases it for half-integer $\tau_{j} / T_{0}$. Accordingly, negative feedback increases the phase diffusion for integer $\tau_{j} / T_{0}$ and suppresses it for half-integer $\tau_{j} / T_{0}$. The efficiency of control for large and small values of the delay time is similar. 
The minimal values of the diffusion constant are:

$-D_{\min } \approx 0.0052$ for $k_{1}=k_{2}=0.15$, attained at $\tau_{1}=\tau_{2} \approx 0.56$

- $D_{\min } \approx 0.0050$ for $k_{1}=-k_{2}=0.15$, attained at $\tau_{1} \approx 0.64, \tau_{2} \approx 0.28$.

Thus, the employment of two feedbacks of different sign allows one to achieve the same level of suppression of the phase diffusion, without nearing to the domains of the chaos crisis.

Notice, the reference values of parameters and variables in the Lorenz system for the classical sets of parameter values are $\sim 10$, meaning $\left|k_{j}\right|=0.15$ is a few percents compared to these values, i.e., the feedback control $k_{j}\left[z\left(t-\tau_{j}\right)-z(t)\right]$ is really weak against the background of the $b z$ - and $x y$-terms.

\subsection{Multistability}

Generally, a sufficiently strong delayed feedback can result in the appearance of locally stable periodic orbits [29] or stabilization of fixed points [1-3]. However, the Pyragas' method (with single or recursive delay feedback $[29,30]$ ) is known to be not stabilizing unstable periodic orbits in the Lorenz system (although the generality of the odd number limitation theorem [23] was later refuted [4, 10], the plain delayed feedback does not stabilize periodic orbits in the Lorenz system). In the case of two delay times, we also did not observe stabilization of periodic orbits.

The stabilization of fixed points of the Lorenz system is possible and poses a danger in our case. The exponential growth rate $\mu$ of linear perturbations is given by the following characteristic equation, which can be derived from the linearization of system $(2.1)-(2.3)$ near $\left\{x_{0}=y_{0}= \pm \sqrt{b(r-1)}, z_{0}=r-1\right\}$ :

$$
\mu(\mu-\sigma-1)\left(\mu+b+k_{1}\left(1-e^{-\mu \tau_{1}}\right)+k_{2}\left(1-e^{-\mu \tau_{2}}\right)\right)+b(r-1)(\mu-2 \sigma)=0 .
$$

This equation is a trancendental one, and the results of its numeric solving are provided in Figures $1 \mathrm{~b}$ and 2 , where the real part of $\mu, \lambda_{\text {st }} \equiv \operatorname{Re} \mu$, is plotted in the bottom panels.

\section{Stochastic oscillators}

It is problematic to construct some general analytical description for the deterministic chaotic systems even approximately. However, if we look solely at the specific characteristic of the regularity — phase diffusion some intuition can be gained within the framework of the phase reduction. With this reduction, the irregularity of the phase growth and amplitude fluctuations from one turnover to another can be modelled as a noise (e.g., some aspects of the representation of deterministic chaotic oscillations by noise were addressed in [24]).

While the stochastic phase description for deterministic chaotic oscillators is only an approximation, this description is mathematically rigorous for limit-cycle oscillators subject to noise. These systems are not least wide-spread in nature and technology than the deterministic chaotic oscillators; and the necessity for control of the precision of their oscillations is even more important and natural.

Let us consider noisy Van der Pol oscillator with two dissipative delayed feedback terms as an example;

$$
\ddot{x}-\mu\left(1-x^{2}\right) \dot{x}+\Omega_{0}^{2} x=k_{1}\left[\dot{x}\left(t-\tau_{1}\right)-\dot{x}(t)\right]+k_{2}\left[\dot{x}\left(t-\tau_{2}\right)-\dot{x}(t)\right]+\varepsilon \zeta(t)
$$

where $\mu>0$ is the bifurcation parameter, $\Omega_{0}$ is the natural frequency of linear oscillations, $k_{j}$ and $\tau_{j}$ are the feedback coefficients and delay times, respectively; $\varepsilon$ is the noise strength, $\zeta(t)$ is the normalized white Gaussian noise: $\left\langle\zeta(t) \zeta\left(t^{\prime}\right)\right\rangle=2 \delta\left(t-t^{\prime}\right),\langle\zeta(t)\rangle=0$. 


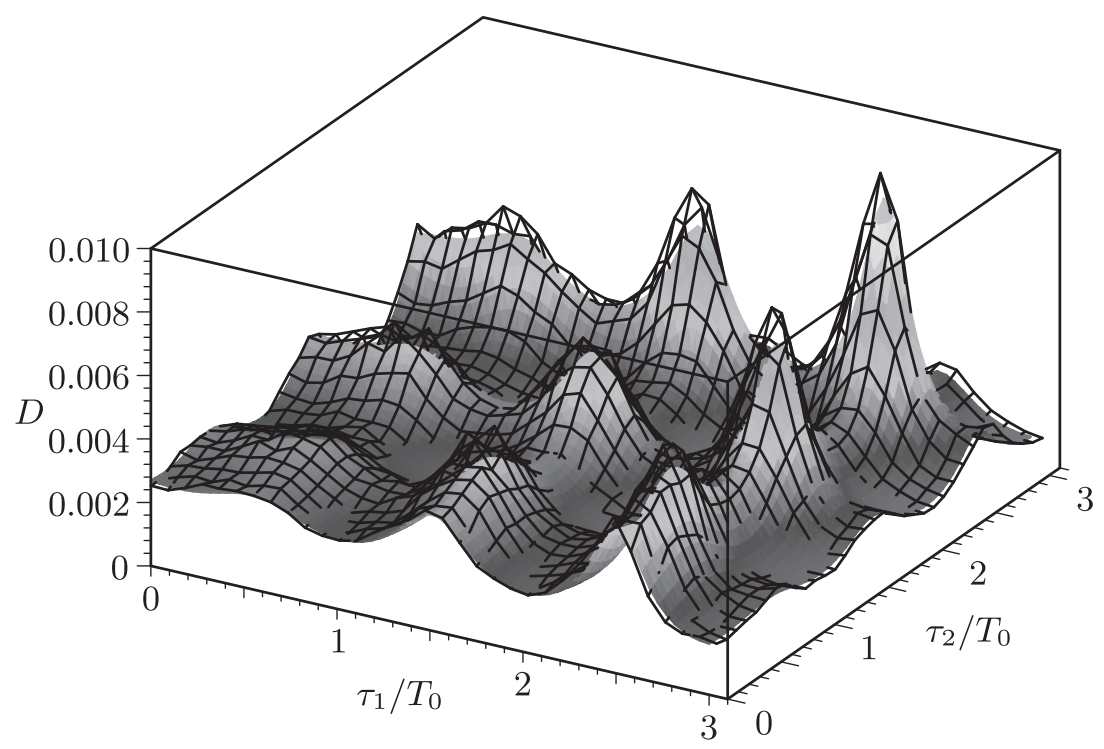

Figure 3. Coherence of noisy Van der Pol oscillator (3.1) subject to two delayed feedbacks with $k_{1}=k_{2}=0.03$, the noise strength $\varepsilon=0.1$; the oscillation period of the noise- and controlfree system for $\mu=0.7, \Omega_{0}=1$ is $T_{0} \approx 6.61$. The results of the direct numerical simulation are plotted with the wireframe; the results of the analytical theory (3.23)-(3.24) with the Gaussian approximation are plotted with the gray nontransparent surface.

\subsection{Phase reduction}

One can introduce "slow" amplitude and "fast" phase variables, $\rho$ and $\varphi: x=\rho \cos \varphi, \dot{x}=y=-\rho \Omega_{0} \sin \varphi$. With the latter relation between $(\rho, \varphi)$ and $(x, y)$, one finds

$$
\dot{\rho} \Omega_{0}=\dot{x} \Omega_{0} \cos \varphi-\dot{y} \sin \varphi, \quad \rho \Omega_{0} \dot{\varphi}=-\dot{x} \Omega_{0} \sin \varphi-\dot{y} \cos \varphi .
$$

Substituting $\dot{x}=y$ and $\dot{y}=\ddot{x}$ from equation (3.1) into the latter equations, one can obtain

$$
\begin{aligned}
\dot{\rho}= & \mu\left(\rho \sin ^{2} \varphi-\frac{\rho^{3}}{4} \sin ^{2} 2 \varphi\right)+k_{1} \sin \varphi\left(\rho_{\tau_{1}} \sin \varphi_{\tau_{1}}-\rho \sin \varphi\right)+k_{2} \sin \varphi\left(\rho_{\tau_{2}} \sin \varphi_{\tau_{2}}-\rho \sin \varphi\right)-\frac{\varepsilon}{\Omega_{0}} \sin \varphi \zeta(t), \\
\dot{\varphi}= & \Omega_{0}+\mu\left[\left(\frac{1}{2}-\frac{\rho^{2}}{4}\right) \sin 2 \varphi-\frac{\rho^{2}}{8} \sin 4 \varphi\right]+k_{1} \cos \varphi\left(\frac{\rho_{\tau_{1}}}{\rho} \sin \varphi_{\tau_{1}}-\sin \varphi\right)+k_{2} \cos \varphi\left(\frac{\rho_{\tau_{2}}}{\rho} \sin \varphi_{\tau_{1}}-\sin \varphi\right) \\
& -\frac{\varepsilon}{\rho \Omega_{0}} \cos \varphi \zeta(t)
\end{aligned}
$$

where subscripts $\tau_{1}$ and $\tau_{2}$ indicate the delayed variable values $\varphi\left(t-\tau_{1}\right)$ and $\varphi\left(t-\tau_{2}\right)$, etc.

For a weak feedback and noise, $k_{j} \sim \varepsilon^{2} \ll \mu \ll \Omega_{0}$, variables $\rho(t)$ and $\psi(t)=\varphi(t)-\Omega_{0} t$ are slow against the background of $\varphi(t)=\Omega_{0} t+\psi(t)$, and one can perform the rigorous Bogolyubov-Krylov averaging procedure [6]. After averaging over fast rotations of $\varphi$ for $k_{j} \sim \varepsilon^{2} \ll \mu \ll \Omega_{0}$, equation (3.2) yields

$$
\dot{\rho} \approx \mu\left(\frac{\rho}{2}-\frac{\rho^{3}}{8}\right)
$$




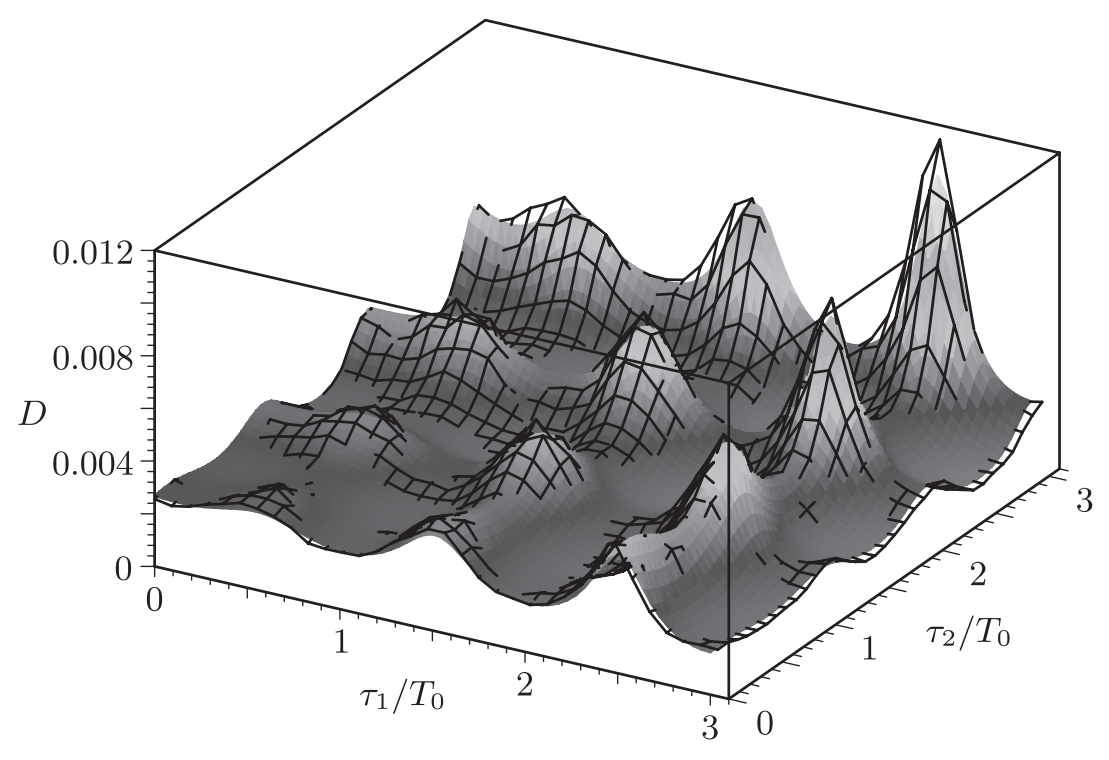

Figure 4. Coherence of noisy Van der Pol oscillator (3.1) subject to two delayed feedbacks with $k_{1}=-k_{2}=0.03, \mu=0.7, \Omega_{0}=1, \varepsilon=0.1$; see the caption for Figure 3 for details.

i.e., the amplitude tends to an approximately constant value, $\rho(t) \approx 2$. In its turn, equation (3.3) can be recast as

$$
\dot{\varphi}(t) \approx \Omega_{0}+\frac{k_{1}}{2} \sin \left[\varphi\left(t-\tau_{1}\right)-\varphi(t)\right]+\frac{k_{2}}{2} \sin \left[\varphi\left(t-\tau_{2}\right)-\varphi(t)\right]-\frac{\varepsilon}{2 \Omega_{0}} \cos \Omega_{0} t \zeta(t) .
$$

The multiplicative white noise $\zeta(t)$ generally creates the Stratonovich drift [12]; for equation system (3.2)-(3.3), the drift term in $\dot{\varphi} \operatorname{reads}\left(-\frac{\varepsilon}{\Omega_{0}} \sin \varphi\right) \frac{\partial}{\partial \rho}\left(-\frac{\varepsilon}{\rho \Omega_{0}} \cos \varphi\right)+\left(-\frac{\varepsilon}{\rho \Omega_{0}} \cos \varphi\right) \frac{\partial}{\partial \varphi}\left(-\frac{\varepsilon}{\rho \Omega_{0}} \cos \varphi\right)=-\frac{\varepsilon^{2}}{\rho^{2} \Omega_{0}^{2}} \sin 2 \varphi$. After averaging over fast rotations of $\varphi$, this term vanishes to the leading order of the Bogolyubov-Krylov expansion and, thus, is not presented in equation (3.4).

Further, on the time scale of slow dynamics of the difference $\left[\varphi\left(t-\tau_{j}\right)-\varphi(t)\right]$, not only $\zeta(t)$ but also $\cos \Omega_{0} t$ is fast oscillating. Hence, the signal $\cos \Omega_{0} t \zeta(t)$ in (3.4), which is $\delta$-correlated, since $\left\langle\cos \left(\Omega_{0} t\right) \zeta(t) \cos \left(\Omega_{0} t^{\prime}\right) \zeta\left(t^{\prime}\right)\right\rangle=$ $2\left\langle\cos ^{2} \Omega_{0} t\right\rangle \delta\left(t-t^{\prime}\right)=\delta\left(t-t^{\prime}\right)$, can be represented by a new effective $\delta$-correlated noise of a halved intensity. We can rewrite equation (3.4) as

$$
\dot{\varphi}(t) \approx \Omega_{0}+\frac{k_{1}}{2} \sin \left[\varphi\left(t-\tau_{1}\right)-\varphi(t)\right]+\frac{k_{2}}{2} \sin \left[\varphi\left(t-\tau_{2}\right)-\varphi(t)\right]+\frac{\varepsilon}{2 \sqrt{2} \Omega_{0}} \xi(t),
$$

where we introduced an effective noise signal $\xi(t)$, which is a normalized white Gaussian noise: $\left\langle\xi(t) \xi\left(t^{\prime}\right)\right\rangle=$ $2 \delta\left(t-t^{\prime}\right),\langle\xi(t)\rangle=0$.

Generally, the provided "simple" procedure of the derivation of equation (3.5) from (3.2)-(3.3) becomes very subtle in the presence of a white noise, and the consideration of the Stratonovich drift term, where it is not negligible, can be insufficiently rigorous. The derivation should be generally performed via the multiple scale expansion for the Fokker-Planck equation (e.g., see [8, 15] or [34]); importantly, for such a derivation, one must strictly use the genuine phase variable, but not a protophase [8]. The Stratonovich drift, if it does not vanish after averaging, merely results in the average frequency bias $[19,35,36]$ and does not make the phase equation more complex [15]. In our case the rigorous general derivation yields the same equation (3.5). 


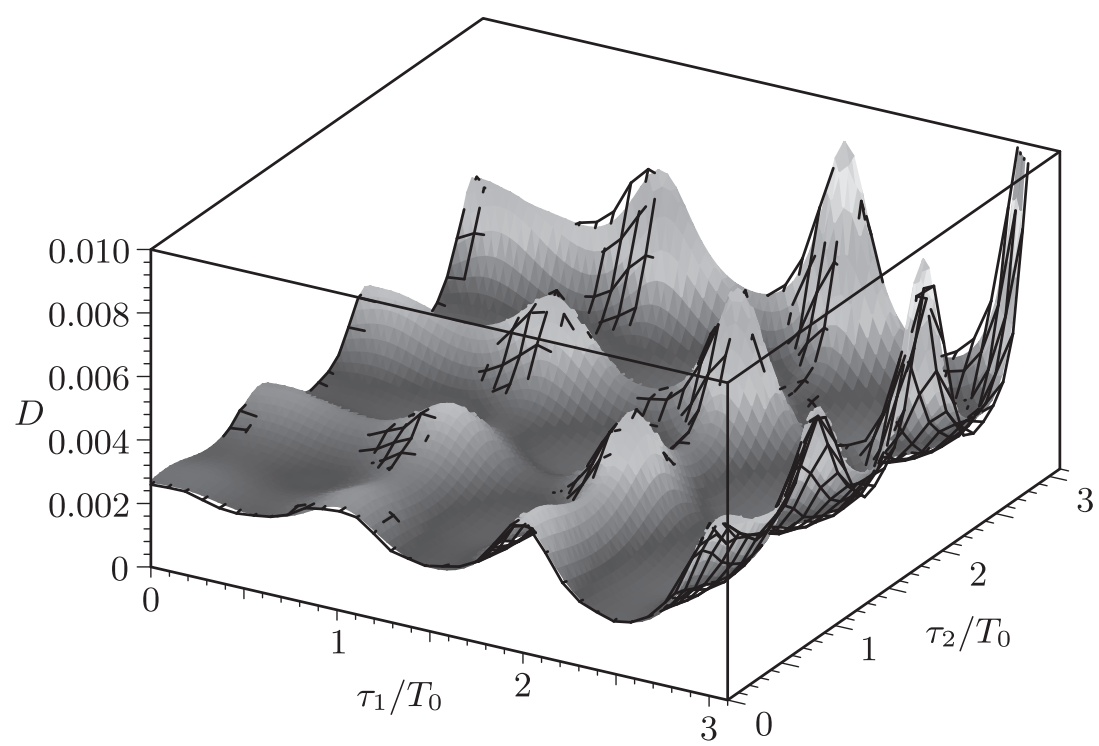

Figure 5. Coherence of noisy Van der Pol oscillator (3.1) subject to two delayed feedbacks with negative $k_{1}=k_{2}=-0.03, \mu=0.7, \Omega_{0}=1, \varepsilon=0.1$; see the caption for Figure 3 for details.

\subsection{Phase diffusion constant}

Let us calculate the phase diffusion constant for the phase reduction equation (3.5). For the deviations of the instantaneous phase growth rate $v$ from $\Omega \equiv\langle\dot{\varphi}\rangle, \dot{\varphi}=\Omega+v,\langle v\rangle=0$, one can recast equation (3.5) as

$$
v(t)=\Omega_{0}-\Omega+\frac{k_{1}}{2} \sin \left(-\Omega \tau_{1}-\int_{0}^{\tau_{1}} v(t-s) \mathrm{d} s\right)+\frac{k_{2}}{2} \sin \left(-\Omega \tau_{2}-\int_{0}^{\tau_{2}} v(t-s) \mathrm{d} s\right)+\frac{\varepsilon}{2 \sqrt{2} \Omega_{0}} \xi(t) .
$$

Similarly to $[17,18]$, we calculate the phase diffusion constant

$$
D=\int_{-\infty}^{+\infty}\langle v(t) v(t+\tau)\rangle \mathrm{d} \tau
$$

from equation (3.6).

With correlation functions

$$
V_{v v}(\tau) \equiv\langle v(t) v(t+\tau)\rangle, \quad V_{v \xi}(\tau) \equiv\langle v(t) \xi(t+\tau)\rangle, \quad V_{\xi \xi}(\tau) \equiv\langle\xi(t) \xi(t+\tau)\rangle
$$

one can write down the average of equation (3.6), the average of its product with $\xi(t+\tau)$ and the average of its product with $v(t+\tau)$ :

$$
0=\Omega_{0}-\Omega-\frac{k_{1}}{2} \sin \Omega \tau_{1}\left\langle\cos \int_{0}^{\tau_{1}} v(t-s) \mathrm{d} s\right\rangle-\frac{k_{2}}{2} \sin \Omega \tau_{2}\left\langle\cos \int_{0}^{\tau_{2}} v(t-s) \mathrm{d} s\right\rangle
$$




$$
\begin{aligned}
V_{v \xi}(\tau) & =-\frac{k_{1}}{2} \sin \Omega \tau_{1}\left\langle\xi(t+\tau) \cos \int_{0}^{\tau_{1}} v(t-s) \mathrm{d} s\right\rangle-\frac{k_{1}}{2} \cos \Omega \tau_{1}\left\langle\xi(t+\tau) \sin \int_{0}^{\tau_{1}} v(t-s) \mathrm{d} s\right\rangle \\
& -\frac{k_{2}}{2} \sin \Omega \tau_{1}\left\langle\xi(t+\tau) \cos \int_{0}^{\tau_{2}} v(t-s) \mathrm{d} s\right\rangle-\frac{k_{2}}{2} \cos \Omega \tau_{1}\left\langle\xi(t+\tau) \sin \int_{0}^{\tau_{2}} v(t-s) \mathrm{d} s\right\rangle+\frac{\varepsilon V_{\xi \xi}(\tau)}{2 \sqrt{2} \Omega_{0}},(3.9) \\
V_{v v}(\tau) & =-\frac{k_{1}}{2} \sin \Omega \tau_{1}\left\langle v(t+\tau) \cos \int_{0}^{\tau_{1}} v(t-s) \mathrm{d} s\right\rangle-\frac{k_{1}}{2} \cos \Omega \tau_{1}\left\langle v(t+\tau) \sin \int_{0}^{\tau_{1}} v(t-s) \mathrm{d} s\right\rangle \\
& -\frac{k_{2}}{2} \sin \Omega \tau_{1}\left\langle v(t+\tau) \cos \int_{0}^{\tau_{2}} v(t-s) \mathrm{d} s\right\rangle-\frac{k_{2}}{2} \cos \Omega \tau_{1}\left\langle v(t+\tau) \sin \int_{0}^{\tau_{2}} v(t-s) \mathrm{d} s\right\rangle+\frac{\varepsilon V_{v \xi}(-\tau)}{2 \sqrt{2} \Omega_{0}} .
\end{aligned}
$$

The average values of nonlinear terms in (3.8)-(3.10) can be calculated either with the linear-in-noise approximation or with the assumption of a Gaussian distribution for $v(t)$. In the linearized theory, the fluctuations of $v$ for a Gaussian noise are Gaussian; therefore, the first approximation is a particular case of the second. Below we employ the Gaussian approximation which can be accurate far beyond the applicability domain of the linear approximation.

For zero-mean Gaussian $x$ and $y$, one can calculate $\langle\cos x\rangle=e^{-\left\langle x^{2}\right\rangle / 2}$ and also employ the Furutsu-Novikov formula $[11,25]$

$$
\langle x F(y)\rangle=\left\langle F^{\prime}(y)\right\rangle\langle x y\rangle
$$

Hence, equation (3.8) acquires the following form:

$$
\Omega=\Omega_{0}-\frac{k_{1}}{2} e^{-R_{1}} \sin \Omega \tau_{1}-\frac{k_{2}}{2} e^{-R_{2}} \sin \Omega \tau_{2}
$$

where

$$
R_{j} \equiv \frac{1}{2}\left\langle\left(\int_{0}^{\tau_{j}} v(t-s) \mathrm{d} s\right)^{2}\right\rangle=\frac{1}{2} \int_{0}^{\tau_{j}} \mathrm{~d} s_{1} \int_{0}^{\tau_{j}} \mathrm{~d} s_{2}\left\langle v\left(t-s_{1}\right) v\left(t-s_{2}\right)\right\rangle=\int_{0}^{\tau_{j}} \mathrm{~d} s\left(\tau_{j}-s\right) V_{v v}(s)
$$

In its turn, the terms $\langle\xi(t+\tau) \cos [\ldots]\rangle=\langle v(t+\tau) \cos [\ldots]\rangle=0$, while $\langle\xi(t+\tau) \sin [\ldots]\rangle=\langle\cos [\ldots]\rangle\langle\xi(t+\tau)[\ldots]\rangle$, etc.; therefore, equations (3.9)-(3.10) acquire the following form:

$$
\begin{aligned}
& V_{v \xi}(\tau)=-\frac{k_{1}}{2} e^{-R_{1}} \cos \Omega \tau_{1} \int_{0}^{\tau_{1}} V_{v \xi}(s+\tau) \mathrm{d} s-\frac{k_{2}}{2} e^{-R_{2}} \cos \Omega \tau_{2} \int_{0}^{\tau_{2}} V_{v \xi}(s+\tau) \mathrm{d} s+\frac{\varepsilon V_{\xi \xi}(\tau)}{2 \sqrt{2} \Omega_{0}} \\
& V_{v v}(\tau)=-\frac{k_{1}}{2} e^{-R_{1}} \cos \Omega \tau_{1} \int_{0}^{\tau_{1}} V_{v v}(s+\tau) \mathrm{d} s-\frac{k_{2}}{2} e^{-R_{2}} \cos \Omega \tau_{2} \int_{0}^{\tau_{2}} V_{v v}(s+\tau) \mathrm{d} s+\frac{\varepsilon V_{v \xi}(-\tau)}{2 \sqrt{2} \Omega_{0}} .
\end{aligned}
$$


Autocorrelation functions $V_{v v}(\tau)$ and $V_{\xi \xi}(\tau)$ are related to the power spectral densities of $v(t)$ and $\xi(t)$ via Fourier transforms [12]:

$$
S_{v v}(\omega)=\frac{1}{2 \pi} \int_{-\infty}^{+\infty} V_{v v}(\tau) e^{-i \omega \tau} \mathrm{d} \tau, \quad S_{\xi \xi}(\omega)=\frac{1}{2 \pi} \int_{-\infty}^{+\infty} V_{\xi \xi}(\tau) e^{-i \omega \tau} \mathrm{d} \tau .
$$

Therefore, it is convenient to consider equations (3.13) and (3.14) in Fourier space, adopting $V_{x y}(\tau) \equiv$ $\int_{-\infty}^{+\infty} S_{x y}(\omega) e^{i \omega \tau} \mathrm{d} \omega:$

$$
\begin{aligned}
& S_{v \xi}(\omega)=-\frac{k_{1}}{2} e^{-R_{1}} \cos \Omega \tau_{1} \frac{e^{i \omega \tau_{1}}-1}{i \omega} S_{v \xi}(\omega)-\frac{k_{2}}{2} e^{-R_{2}} \cos \Omega \tau_{2} \frac{e^{i \omega \tau_{2}}-1}{i \omega} S_{v \xi}(\omega)+\frac{\varepsilon S_{\xi \xi}(\omega)}{2 \sqrt{2} \Omega_{0}}, \\
& S_{v v}(\omega)=-\frac{k_{1}}{2} e^{-R_{1}} \cos \Omega \tau_{1} \frac{e^{i \omega \tau_{1}}-1}{i \omega} S_{v v}(\omega)-\frac{k_{2}}{2} e^{-R_{2}} \cos \Omega \tau_{2} \frac{e^{i \omega \tau_{2}}-1}{i \omega} S_{v v}(\omega)+\frac{\varepsilon S_{v \xi}(-\omega)}{2 \sqrt{2} \Omega_{0}} .
\end{aligned}
$$

Substituting $S_{v \xi}(-\omega)$ from equation (3.16) into equation (3.17) with the obvious symmetry property $S_{\xi \xi}(\omega)=$ $S_{\xi \xi}(-\omega)$, one finds

$$
S_{v v}(\omega)=\frac{\varepsilon^{2} S_{\xi \xi}(\omega)}{8 \Omega_{0}^{2}\left|1+\frac{k_{1}}{2} e^{-R_{1}} \cos \Omega \tau_{1} \frac{e^{i \omega \tau_{1}}-1}{i \omega}+\frac{k_{2}}{2} e^{-R_{2}} \cos \Omega \tau_{2} \frac{e^{i \omega \tau_{2}-1}}{i \omega}\right|^{2}} .
$$

For a $\delta$-correlated noise $\xi, V_{\xi \xi}(\tau)=2 \delta(\tau)$ and $S_{\xi \xi}(\omega)=\pi^{-1}$, and thus

$$
S_{v v}(\omega)=\frac{\varepsilon^{2}}{8 \pi \Omega_{0}^{2}\left|1+\frac{k_{1}}{2} e^{-R_{1}} \cos \Omega \tau_{1} \frac{e^{i \omega \tau_{1}-1}}{i \omega}+\frac{k_{2}}{2} e^{-R_{2}} \cos \Omega \tau_{2} \frac{e^{i \omega \tau_{2}-1}}{i \omega}\right|^{2}} .
$$

Comparing equation (3.7) to equations (3.15), one can see that

$$
D=2 \pi S_{v v}(0)
$$

Hence, equation (3.18) at $\omega=0$ yields

$$
\begin{aligned}
D=\lim _{\omega \rightarrow 0} 2 \pi S_{v v}(\omega) & =\frac{\varepsilon^{2}}{4 \Omega_{0}^{2}\left(1+\frac{1}{2} k_{1} \tau_{1} e^{-R_{1}} \cos \Omega \tau_{1}+\frac{1}{2} k_{2} \tau_{2} e^{-R_{2}} \cos \Omega \tau_{2}\right)^{2}} \\
& =\frac{D_{0}}{\left(1+\frac{1}{2} k_{1} \tau_{1} e^{-R_{1}} \cos \Omega \tau_{1}+\frac{1}{2} k_{2} \tau_{2} e^{-R_{2}} \cos \Omega \tau_{2}\right)^{2}},
\end{aligned}
$$

where $D_{0}=\varepsilon^{2} /\left(4 \Omega_{0}^{2}\right)$ is the diffusion constant in the control-free system.

The yet-to-be-defined ingredient is $R_{1,2}$, which are given in terms of the autocorrelation function by equation (3.12). In spectral form, the integral (3.12) can be recast as

$$
R_{j}=\int_{-\infty}^{+\infty} \frac{1-\cos \omega \tau_{j}}{\omega^{2}} S_{v v}(\omega) \mathrm{d} \omega
$$


where we have used that $S_{v v}(\omega)$ is an even function. Unfortunately, the analytical calculation of this integral with (3.19) is not possible. For an approximate calculation of $R_{j}$, we can replace $S_{v v}(\omega)$ with $S_{v v}(0)$ and find

$$
R_{j} \approx \pi \tau_{j} S_{v v}(0)=\frac{D \tau_{j}}{2}
$$

Physically, this approximation corresponds to a diffusion approximation for $R(\tau)=\left\langle[\varphi(t)-\varphi(t-\tau)]^{2}\right\rangle / 2$ (see Eq. (3.12)). Indeed, by definition $\lim _{\tau \rightarrow \infty} \frac{\left\langle[\varphi(t)-\varphi(t-\tau)]^{2}\right\rangle}{\tau}=D$; if one approximately employs the same law for a finite $\tau$, then $R(\tau) \approx D \tau / 2$, which is equation (3.22). This approximation is practically not less accurate than the linear-in-noise approximation and the linear-in-feedback one; additionally, it works well for a strong noise [18].

Finally, we arrive to a transcendental equation system for $\Omega(3.12)$ and $D(3.20)$ :

$$
\begin{aligned}
& \Omega=\Omega_{0}-\frac{k_{1}}{2} e^{-D \tau_{1} / 2} \sin \Omega \tau_{1}-\frac{k_{2}}{2} e^{-D \tau_{2} / 2} \sin \Omega \tau_{2}, \\
& D=\frac{D_{0}}{\left(1+\frac{1}{2} k_{1} \tau_{1} e^{-D \tau_{1} / 2} \cos \Omega \tau_{1}+\frac{1}{2} k_{2} \tau_{2} e^{-D \tau_{2} / 2} \cos \Omega \tau_{2}\right)^{2}} .
\end{aligned}
$$

In Figures 3-5, the dependence of the diffusion constant on delay times is presented for $k_{1}=k_{2}>0, k_{1}=-k_{2}$ and $k_{1}=k_{2}<0$, respectively. As one should expect with equations (3.23)-(3.24), positive feedback suppresses the phase diffusion for integer $\tau_{j} / T_{0}$ and increases it for half-integer $\tau_{j} / T_{0}$; negative feedback increases the phase diffusion for integer $\tau_{j} / T_{0}$ and suppresses it for half-integer $\tau_{j} / T_{0}$. In contrast to the case of chaotic oscillators, where the control efficiency for small and large $\tau_{j}$ is similar (see Figs. 1 and 2), the efficiency of control for stochastic oscillators grows with $\tau_{j}$ almost linearly. One should expect this property from equation (3.24), where the diffusion constant $D$ depends of the product $k_{j} \tau_{j}$; strictly speaking, this property is approximate, because $D$ also depends on $\Omega$, and $k_{j}$ appear in equation (3.23) without factor $\tau_{j}$.

One can see that the analytical theory (3.23)-(3.24), which is derived on the basis of the Gaussian approximation for $v$, works reasonably well even where the effect of feedback is strong enough to suppress/increase the phase diffusion constant by one order of magnitude. Practically, the Gaussian approximation can be employed as long as the probability density of the relevant random variable remains unimodal. The noise-free system with delayed feedback can exhibit multistability (see equation (3.11) with $R_{j}=0$ ) for $k_{1} \tau_{1}+k_{2} \tau_{2}>2$ (see [17] for the stability analysis of a system with single delay). In the case of multistability of the noise-free system, the probability density of $v$ in the presence of noise possesses several peaks. The phase diffusion in this case can be extremely strong [28] and the system demonstrates nontrivial behaviour [7, 28], which is however beyond the scope of this paper.

\section{CONCLUSiOn}

We have studied the effect of a feedback with two delay times on coherence of chaotic oscillations, which is quantified by the phase diffusion constant $D$, and revealed that the diffusion constant in the Lorenz system can vary by 2-3 orders of magnitude. The analysis of the chaotic properties of the system and the stability of nonchaotic regimes (fixed points) suggests that the employment of two delay feedback control can be beneficial. For instance, in the case of different signs of the coefficients, $k_{1}=-k_{2}$, one can achieve a suppression (or increase) of the phase diffusion by one order of magnitude more than with a single delay feedback [17], without destroying the chaotic regime.

The intuition on the effect of multiple delay feedback on chaotic systems can be earned with the analytical theory constructed within the framework of the phase reduction. The phase model is a rough simplification for chaotic oscillators, but is rigorous for stochastic oscillators. For the latter-limit-cycle oscillators subject to noise - the effect is less nontrivial, but these systems are even more widespread in nature and technology than the chaotic oscillators. The theory yields a self-contained equation system for the average frequency and 
the phase diffusion constant (3.23)-(3.24). As one should expect for both chaotic and stochastic oscillators, the positive feedback suppresses the phase diffusion for integer $\tau_{j} / T_{0}$ and increases it for half-integer $\tau_{j} / T_{0}$, while the negative one increases $D$ for integer $\tau_{j} / T_{0}$ and suppresses $D$ for half-integer $\tau_{j} / T_{0}$. Remarkably, however,

- the control efficiency grows almost linearly with delay time $\tau_{j}$ for stochastic oscillators (Figs. 3-5), while for chaotic oscillators it is similar for small and large $\tau_{j}$ (see top panels of Figs. $1 \mathrm{~b}$ and 2);

- the effect of control for chaotic oscillators seems to be exponentially strong (as it was also observed in [17]), and one can increase/suppress the phase diffusion by two orders of magnitude by means of a relatively weak feedback.

Acknowledgements. The work has been supported by the Russian Science Foundation (Grant no. 19-42-04120).

\section{REFERENCES}

[1] A. Ahlborn and U. Parlitz, Stabilizing Unstable Steady States Using Multiple Delay Feedback Control. Phys. Rev. Lett. 93 (2004) 264101.

[2] A. Ahlborn and U. Parlitz, Controlling dynamical systems using multiple delay feedback control. Phys. Rev. E 72 (2005) 016206.

[3] A. Ahlborn and U. Parlitz, Laser stabilization with multiple-delay feedback control. Opt. Lett. 31 (2006) $465-467$.

[4] A. Amann and E.W. Hooton, An odd-number limitation of extended time-delayed feedback control in autonomous systems. Phil. Trans. R. Soc. A 371 (2013) 20120463.

[5] S. Boccaletti, E. Allaria and R. Meucci, Experimental control of coherence of a chaotic oscillator. Phys. Rev. E 69 (2004) 066211.

[6] N. Bogoliubov, Asymptotic Methods in the Theory of Non-Linear Oscillations, Gordon \& Breach, Paris (1961).

[7] O. D'Huys, Th. Jungling and W. Kinzel, Stochastic switching in delay-coupled oscillators. Phys. Rev. E 90 (2014) 032918.

[8] A.V. Dolmatova, D.S. Goldobin and A. Pikovsky, Synchronization of coupled active rotators by common noise. Phys. Rev. E 96 (2017) 062204.

[9] V.V. Emel'yanov, N.M. Ryskin and O.S. Khavroshin, Suppression of Self-Modulation in the Self-Oscillator with Delayed Feedback Using the Method for Chaos Control. J. Commun. Tech. Electron. 54 (2009) 685-691.

[10] B. Fiedler, V. Flunkert, M. Georgi, P. Hövel and E. Schöll, Refuting the odd number limitation of time-delayed feedback control. Phys. Rev. Lett. 98 (2007) 114101.

[11] K. Furutsu, On the statistical theory of electromagnetic waves in a fluctuating media. J. Res. Natl. Bur. Stand. D 67 (1963) 303-323.

[12] C.W. Gardiner, Handbook of Stochastic Methods. Springer, Berlin (1983).

[13] D.S. Goldobin, Anharmonic resonances with recursive delay feedback. Phys. Lett. A 375 (2011) 3410-3414.

[14] D.S. Goldobin, Uncertainty principle for control of ensembles of oscillators driven by common noise. EPJST 223 (2014) 677-685.

[15] D.S. Goldobin and A.V. Dolmatova, Interplay of the mechanisms of synchronization by common noise and global coupling for a general class of limit-cycle oscillators. Commun. Nonlinear Sci. Numer. Simulat. 75 (2019) 94-108.

[16] D.S. Goldobin and L.S. Klimenko, Resonances and multistability in a Josephson junction connected to a resonator. Phys. Rev. E 97 (2018) 022203.

[17] D. Goldobin, M. Rosenblum and A. Pikovsky, Controlling oscillator coherence by delayed feedback. Phys. Rev. E 67 (2003) 061119 .

[18] D. Goldobin, M. Rosenblum and A. Pikovsky, Coherence of noisy oscillators with delayed feedback. Phys. A 327 (2003) $124-128$.

[19] D.S. Goldobin, J.-N. Teramae, H. Nakao and G.B. Ermentrout, Dynamics of Limit-Cycle Oscillator Subject to General Noise. Phys. Rev. Lett. 105 (2010) 154101.

[20] Y.M. Guznov, Y.Y. Danilov, S.V. Kuzikov, Y.V. Novozhilova, A.S. Shevchenko, N.I. Zaitsev and N.M. Ryskin, Megawatt-power Ka-band gyroklystron oscillator with external feedback. Appl. Phys. Lett. 103 (2013) 173505.

[21] B. Kralemann, L. Cimponeriu, M. Rosenblum, A. Pikovsky and R. Mrowka, Uncovering interaction of coupled oscillators from data. Phys. Rev. E 76 (2007) 055201(R).

[22] E.N. Lorenz, Deterministic Nonperiodic Flow. J. Atmos. Sci. 20 (1963) 130-141.

[23] H. Nakajima, On analytical properties of delayed feedback control of chaos. Phys. Lett. A 232 (1997) $207-210$.

[24] H. Nakao, J.-N. Teramae, D.S. Goldobin and Y. Kuramoto, Effective long-time phase dynamics of limit-cycle oscillators driven by weak colored noise. Chaos 20 (2010) 033126.

[25] Y. Novikov, Functionals and the random force method in turbulence theory. Sov. Phys. JETP 20 (1965) $1290-1294$.

[26] A.H. Pawlik and A. Pikovsky, Control of oscillators coherence by multiple delayed feedback. Phys. Lett. A 358 (2006) $181-185$.

[27] A. Pikovsky, M. Rosenblum and J. Kurths, Synchronization: A Universal Concept in Nonlinear Sciences. Cambridge Univ. Press, Cambridge (2001). 
[28] A.V. Pimenova and D.S. Goldobin, Coherence of Noisy Oscillators with Delayed Feedback Inducing Multistability. J. Phys.: Conf. Ser. 681 (2016) 012045.

[29] K. Pyragas, Continuous control of chaos by self-controlling feedback. Phys. Lett. A 170 (1992) 421-428.

[30] K. Pyragas, Control of chaos via extended delay feedback. Phys. Lett. A 206 (1995) 323-330.

[31] N.M. Ryskin and O.S. Khavroshin, Suppressing Self-Modulation Instability in a Delayed Feedback Traveling Wave Tube Oscillator Using Controlling Chaos Technique. IEEE Transactions on Electron Devices 55 (2008) 662-667.

[32] A.E. Samoilova and A. Nepomnyashchy, Feedback control of Marangoni convection in a thin film heated from below. J. Fluid Mech. 876 (2019) 573-590.

[33] A.E. Samoilova and A. Nepomnyashchy, Nonlinear feedback control of Marangoni wave patterns in a thin film heated from below. Physica D: Nonlinear Phenomena 412 (2020) 132627.

[34] R.L. Stratonovich, Topics in the Theory of Random Noise. Gordon and Breach, New York (1967).

[35] J.-N. Teramae, H. Nakao and G.B. Ermentrout, Stochastic Phase Reduction for a General Class of Noisy Limit Cycle Oscillators. Phys. Rev. Lett. 102 (2009) 194102.

[36] K. Yoshimura and K. Arai, Phase Reduction of Stochastic Limit Cycle Oscillators. Phys. Rev. Lett. 101 (2008) 154101. 